\title{
Controversias en torno a los métodos de medición y a las medidas oficiales de la pobreza en la Argentina reciente
}

\author{
Soledad A. Pérez*
}

\begin{abstract}
Resumen
En el presente trabajo se abordan las controversias vinculadas a los métodos de medición y a las medidas oficiales de la pobreza en Argentina, en el periodo 2003-2011, pues las controversias, tanto las teóricas como las metodológicas, suelen tener un papel fundamental en el desarrollo epistemológico. Para ello se distinguen tres periodos: en el primero, entre 2003 y 2005, se entabló una larga polémica centrada en lo metodológico, aunque no exclusivamente, entre la Dirección Nacional de Coordinación de Política Macroeconómica - dependiente del Ministerio de Economía - y el Instituto Nacional de Estadísticas y Censos (INDEC). El segundo tuvo lugar en 2006, y en las controversias, donde se enzarzaron esta vez el INDEC y la Secretaría de Comercio Interior, perdió preponderancia la discusión metodológica relativa a la medición de la pobreza. El último periodo se inició en enero de 2007, momento a partir del cual se incorporaron nuevos actores a las controversias y se modificó el eje de las discusiones, que se desplazó de los métodos, y de los conceptos que se asumen en éstos, a la legitimidad del proceso de medición y de sus resultados.
\end{abstract}

\begin{abstract}
Theoretical as well as methodological controversies often have a fundamental role in epistemic development. This paper addresses the controversies regarding the methods and the official poverty measurements in Argentina between 2003 and 2011. Results point to three different phases: the first one, between 2003 and 2005, when controversies were about methodology and starred the National Macroeconomic Policy Coordinating body and the National Institute for Statistics and Census (INDEC). The second, during 2006, when the dispute was led between the INDEC and the Ministry of Domestic Trade and methodological discussions on poverty lost dominance. The final period began in 2007, when new players joined the controversies and the legitimacy of the measurement results became the focus of discussions.
\end{abstract}

Palabras clave: controversias, matriz, pobreza, mediciones oficiales, métodos, Argentina Key Words: controversies, matrix, poverty, official measurement, methodology, Argentina.

\footnotetext{
* Conicet, Fundación Bariloche.
} 
el presente trabajo abordaremos las controversias vinculadas a los métodos de medición y a las medidas oficiales de la pobreza en Argentina durante el periodo 2003-2011, pues las controversias, tanto las teóricas como las metodológicas, suelen tener un papel fundamental en el desarrollo epistemológico (Enhelhardt, 1981; Dascal, 1995, 2006; Armatte, 2000, 2002, 2004; Nudler, 2009). La adopción de este enfoque obedece a que al estudiar controversias "tenemos los verdaderos argumentos ante los ojos, mientras que si estudiamos únicamente los argumentos vencedores nunca sabremos por qué han perdido los que han sido eliminados" (Armatte, 2000: 18-19).

Estos intercambios requieren la existencia de al menos dos personas que mutuamente se dirijan y confronten opiniones, argumentos, teorías, etc. Sin embargo, pese a su estructura, las controversias deben ser consideradas cuasi diálogos, porque la forma que asumen se vincula a la presencia de una presunta platea, que motiva la elección de distintas maniobras pragmáticas por parte de los oponentes (Dascal, 2006). Sumadas al requisito mínimo de bilateralidad, entre las características salientes de este tipo de intercambios encontramos la apertura y la capacidad expansiva, ya que frente a cada controversia siempre existe la posibilidad de que se amplíen tanto el espectro de actores involucrados en las mismas como los temas asociados y las disciplinas intervinientes, lo cual se enlaza con otro de sus atributos: el de ser imprevisibles.

Coincidiendo con el valor que los autores citados previamente asignan a la confrontación de ideas como impulsoras del pensamiento y el conocimiento, y sirviéndose de su concepto de espacios controversiales, Nudler se orienta a determinar de qué modo se producen los avances y estancamientos dentro de la historia intelectual, qué factores intervienen y qué formas adquiere dicha evolución, en términos de rupturas y continuidades, esclareciendo problemas vinculados a la creación y al cambio de significado. Esta propuesta tiene la particularidad de que considera las controversias, no como elementos aislados, sino formando parte de un conjunto dinámico y articulado de elementos al que dan cohesión los problemas o temas en torno a los cuales surgen las teorías, las metodologías y las tradiciones de investigación, en las que se enrolan los participantes en las polémicas. Estos elementos componen la región visible del espacio controversial, es decir, el foco. Los presupuestos compartidos por los participantes en una controversia - presupuestos difícilmente perceptibles a menudo - son condición indispensable para que pueda darse un debate racional. De no existir este mínimo nivel de acuerdo, 
la relación que podría entablarse oscilaría entre la indiferencia o el conflicto, entendido éste último como confrontación no racional. Estos acuerdos tácitos pueden concernir tanto a cuestiones metodológicas como teóricas.

Cada uno de los elementos mencionados, es decir, los problemas y las controversias (tanto genuinas como ficcionales), ${ }^{1}$ integran el foco del espacio controversial y tienen como telón de fondo y condición de posibilidad a los supuestos compartidos que integran el terreno común. Entre estos elementos existe una relación de interacción, de modo que el cambio en uno repercute sobre los otros, extendiéndose a toda la estructura del espacio controversial. Esta influencia mutua entre los componentes de dicho espacio, nos muestra que no son estructuras fijas; responden a distintos procesos de cambio que llevan a la creación de nuevos conceptos, o a dar un nuevo significado a los ya existentes, cuando se cuestiona alguno de los supuestos compartidos sobre los que se construyó el espacio controversial (refocalizaciones), de modo que el foco pasa a estar constituido por algún punto que resultaba previamente incuestionable; y también se registran modificaciones menores cuando se producen cambios en los focos y permanece inalterado el suelo de los supuestos compartidos.

En este ámbito, y a fin de analizar el proceso controversial en torno a las mediciones y medidas oficiales de la pobreza en la Argentina reciente, en principio encuadraremos de manera general las controversias en el marco de las discusiones que se dan en torno a la medición de la pobreza a nivel internacional, para posteriormente centrarnos en las controversias que son el objeto de este trabajo. Respecto de estas últimas, distinguiremos tres periodos: en el primero, entre 2003 y 2005, se desarrollaron controversias entre la Dirección Nacional de Coordinación de Política Macroeconómica, dependiente del Ministerio de Economía, y el Instituto Nacional de Estadísticas y Censos; el segundo se dio a lo largo de 2006 y las controversias se entablaron entre el INDEC y la Secretaría de Comercio Interior. El último periodo se inició en enero de 2007 y aún no ha culminado.

\section{Enmarcando las controversias}

Tanto la definición como la medición de la pobreza, involucran un sinnúmero de discusiones. Si bien existe consenso para asociar el fenómeno a situaciones de privación o carencia, no es fácil alcanzar acuerdos respecto

1 Al hablar de controversias ficcionales, nos referimos a las discusiones que se entablan con autores no contemporáneos y en las que, por lo mismo, es imposible obtener una respuesta. 
a los elementos cuya carencia permite identificar una situación como de pobreza, ni tampoco respecto de los grados o matices en que ciertas privaciones podrían hacer ingresar a un individuo u hogar en esta categoría.

Tal como ha señalado Sen (1976) en sus tempranos trabajos, el proceso de medición de la pobreza atraviesa dos instancias: la identificación ${ }^{2}$ y la agregación. La primera remite a la definición de los criterios que permitirán distinguir a los pobres de quienes no lo son, mientras que el momento de agregación da cuenta del modo en que se combinan los datos en un indicador general de la pobreza.

La etapa de identificación conduce a problemas similares a los de las series de sorites o paradoja del montón. Estas series aluden a la vaguedad intrínseca de ciertos conceptos y a las dificultades que se derivan de la búsqueda de un límite o punto de corte entre objetos que poseen o no una propiedad; si se van sumando granos de arena, ¿en qué momento, a partir de qué grano es posible afirmar que estamos frente a un montón

2 En la actualidad existen tres enfoques principales para identificar a los pobres en un contexto multidimensional: el enfoque unidimensional, a través del cual se combinan los distintos indicadores de bienestar en una sola variable agregada, y una persona es identificada como pobre cuando la variable cae debajo de determinada línea de corte. Este método toma en cuenta privaciones dimensionales, pero sólo en cuanto afectan a un indicador agregado. Como sostienen Alkire y Foster (2008), existe un margen escaso para evaluar las privaciones dimensionales en sí mismas, lo que constituye una característica esencial para el enfoque multidimensional. Esta forma de identificación unidimensional implica varias presunciones que tornan el método criticable; como sostienen Alkire y Foster (2008), fundamentalmente la dificultad conceptual de ver a la pobreza multidimensional a partir de una lente unidimensional, es la pérdida de información sobre la deficiencia específica en cada dimensión, puesto que la agregación antes de la identificación convierte a los desempeños dimensionales en uno solo, sin considerar las líneas de corte específicas de cada dimensión. Desde esta perspectiva, se considera pobre a una persona si el valor monetario del conjunto de desempeños está por debajo del costo del conjunto de mercancías al que se presume debería acceder para no caer en la pobreza. El método de identificación de unión, evita esta dificultad, ya que considera que una persona es pobre multidimensionalmente si experimenta privaciones en alguna de las dimensiones consideradas. Aquí el peligro es sobreestimar la pobreza, o incluir a individuos u hogares que podrían no ser pobres para algunos intérpretes. Otra dificultad que presenta este método, es que puede no distinguir a los más pobres entre los pobres, lo que puede implicar desatenderlos; sin embargo, el método de la unión se utiliza en índices como el de Pobreza Humana (IPH) o el de Necesidades Básicas Insatisfechas. El tercer enfoque es el método de la intersección, que exige que una persona registre privaciones en todas las dimensiones para ser considerada pobre. Este enfoque a menudo es criticado justamente por lo contrario del anterior, ya que tiende a subestimar la pobreza. El foco aquí está puesto en los más desposeídos, excluyendo a gran cantidad de personas que pueden experimentar privaciones. La capacidad de este enfoque de identificar pobres disminuye a medida que se incrementa la cantidad de dimensiones, es decir, que la relación entre la posibilidad de que ciertos individuos u hogares sean considerados pobres, es inversamente proporcional a la cantidad de dimensiones consideradas: a más dimensiones, menor cantidad de pobres. Aquí la dificultad es evidente, se corre el riesgo de no considerar como pobres a quienes sufren privaciones. 
de arena? Al trasladar estos interrogantes a la medición de la pobreza, se formulan preguntas como las siguientes: ¿cómo establecer el corte entre los que son pobres y los que no? ¿Cómo establecer el umbral de la pobreza? ¿Cómo y dónde realizar el corte? ¿Qué dimensiones deben ser consideradas? ¿Cómo relacionar los umbrales de cada una de las dimensiones? Si ordenamos a los individuos en función de los ingresos que perciben, y si $\$ 1$ constituye la distancia entre el nivel de ingresos de uno y otro, ¿dónde establecer el límite entre el que es pobre y el que no lo es? ¿Cómo resolver la arbitrariedad presente entre los que se encuentran en posiciones contiguas, prácticamente iguales, cuando se establece una línea de corte?

En los organismos financieros internacionales suelen subestimarse las dificultades que plantean estos interrogantes, y se sostiene que se trata de una definición arbitraria como tantas otras, implícitas en un proceso de investigación; pero la denuncia del fantasma de la arbitrariedad no basta para exorcizarlo. Como señala uno de los protagonistas de estas controversias: "Decir la verdad acerca de la dimensión de la pobreza depende tanto de que tengamos un concepto adecuado [...] como de que tengamos herramientas para evaluar su magnitud empírica" (Reddy: 214). ${ }^{3}$

En este sentido, diversos autores como Sen $(1976,1992,2000)$, Nussbaum (1996, 2003), Alkire y Foster (2010), Reddy y Pogge (2003) y Boltvinik (2005), consideran no sólo arbitrario el establecimiento de un corte sino también simplista, pues reduce el asunto al enfoque monetario, por lo que impulsan una mirada centrada en conceptos que complejicen la comprensión de la pobreza. ${ }^{4}$ En línea con lo anterior, estudios realizados en países desarrollados muestran la débil relación que existe entre ingresos y privación, lo cual demuestra la insuficiencia de identificar a los pobres sólo mediante el ingreso (Nolan y Whelan, 1996).

Esto evidencia la necesidad de adoptar medidas multidimensionales de la pobreza, como la estrategia planteada por Alkire y Foster (2008), Alkire y Santos (2010), o como el Método de Medición Integrada de la Pobreza, que combinando la medición por ingresos con la de Necesidades Básicas

3 Para una reconstrucción de las controversias mantenidas por Pogge y Reddy con Ravallion, quien encabeza la defensa de las mediciones del Banco Mundial, se recomienda consultar How not to count the poor, de Pogge y Reddy (2003), y la respuesta de Ravallion, cuyas referencias se encuentran en la bibliografía general del artículo.

4 Una reconstrucción de las controversias en torno al concepto de pobreza, desde su constitución en objeto de estudio a mediados del siglo XIX hasta la actualidad, se encuentra en La pobreza como espacio controversial. Reflexiones en torno a las dinámicas de cambio conceptual y progreso epistémico, de Soledad A. Pérez, Editorial Académica Española, 2012. 
Insatisfechas (Boltvinik, 1992, 2005), logra en alguna medida reducir las limitaciones de ambos métodos usados aisladamente.

\section{El INDEC vs. la Dirección Nacional de Coordinación de Políticas Macroeconómicas (2003-2005)}

Estas líneas introductorias acerca de las perspectivas contrapuestas en lo relativo a los métodos de medición de la pobreza a nivel internacional, permiten considerar las controversias que se dieron en Argentina en los últimos años, no como un fenómeno aislado, sino inscrito en una trama sumamente compleja en la que se articulan cuestiones teóricas, metodológicas, políticas y económicas.

Cabe agregar que las polémicas que constituyen nuestro objeto de análisis, tuvieron como marco al Instituto Nacional del Estadísticas y Censos, y que los protagonistas de dichas disputas combinaban rasgos de una formación científica o técnica con la pertenencia a la burocracia estatal (Otero, 2006).

Es importante reconocer que el sistema estadístico argentino ha experimentado, a partir de 1960, sucesivos perfeccionamientos, y también ciertos retrocesos puntuales, en la medición de los niveles de bienestar de la población. Ya el censo de 1960 implementó estadísticas con capacidad para definir diagnósticos y estrategias territoriales de intervención social que adquirirían "todo su significado y potencialidad dos décadas más tarde, a partir de la difusión de conceptos como vulnerabilidad y grupos de riesgo" (Otero, 2011: 19). Pese a que conceptos análogos habían existido en el pasado, "las ideas-fuerza de la política social del modelo aperturista, es decir, las de 'focalización' y 'grupos vulnerables' (Torrado, 2006), potenciaron desde entonces el valor informacional de un amplio conjunto de indicadores que el sistema estadístico nacional puso en marcha mucho antes de que su potencial utilidad se convirtiera en acuciante" (Otero, 2011: 19). Así, la medición de las Necesidades Básicas Insatisfechas, ${ }^{5}$ a partir de

5 Las Necesidades Básicas Insatisfechas, según el Censo 2001, fueron definidas a partir de la metodología empleada en La pobreza en Argentina (serie estudios del indec, $\mathrm{N}^{\circ} 1,1984$ ). En este documento, se considera que un hogar tiene sus Necesidades Básicas Insatisfechas si presenta alguna de estas características: hacinamiento (hogares con más de tres personas por cuarto); vivienda (hogares en una vivienda de tipo inconveniente - pieza de inquilinato, vivienda precaria u otro tipo, lo que excluye casa, departamento y rancho-); condiciones sanitarias (hogares que no tengan ningún tipo de retrete); asistencia escolar (hogares que tuvieran algún niño en edad escolar -6 a 12 años - que no asistiera a la escuela); capacidad de subsistencia (hogares 
1980, manifestó la utilidad de las preguntas sobre vivienda para diagnosticar fenómenos emergentes, especialmente la pobreza, tras la instalación del modelo aperturista de la economía con el golpe de Estado de 1976.

Es en este marco de antecedentes que ha de situarse la controversia entre el Instituto Nacional de Estadísticas y Censos y la Dirección Nacional de Coordinación de Políticas Macroeconómicas (DNCPM), organismos dependientes del Ministerio de Economía de la Nación; dicha controversia se desató a partir de la publicación del informe Población indigente y pobre en el total urbano: metodología alternativa, publicado por la segunda de estas instituciones en enero de 2003. En dicho informe, partiendo del enfoque del ingreso, ${ }^{6}$ se daban a conocer mediciones de la pobreza alternativas a las del INDEC.

Este pronunciamiento fue rebatido inmediatamente por dicho Instituto a través de un artículo, publicado en el mes de marzo, donde contrariando a la DNCPM, se destacaba la existencia de "un principio metodológico básico", consistente en que "cuando se dispone de nueva información, no resulta recomendable realizar modificaciones parciales de una metodología sino que deben encararse revisiones integrales" (INDEC, 2003b: 1).

Desde el organismo oficial de estadísticas se recomendaba que los cambios para medir la pobreza se realizaran en el marco de una actualización comprensiva, como la que había emprendido el INDEC a principios de la década, involucrando la actualización tanto de los bienes que integran la Canasta Básica de Alimentos, como de la relación inicial entre los gastos alimentarios y no alimentarios de la población de referencia, a partir de una nueva fuente de información como la Encuesta Nacional de Gastos 1996/1997.

Cabe aclarar que ambas metodologías, la del INDEC y la de la DNCPM, utilizaban la misma Canasta Básica; es decir: en términos de Nudler, ambos organismos compartían ciertos presupuestos que actuaban como telón de fondo y condición de posibilidad de la controversia suscitada por el criterio utilizado por la DNCPM para valorizar dicha Canasta, ya que proponía usar "un promedio entre los precios IPC (Índice de Precios al Consumidor) y los

que tuvieran cuatro o más personas por miembro ocupado, y además cuyo jefe no haya completado el tercer grado de escolaridad primaria).

6 El enfoque del ingreso consiste en calcular el ingreso mínimo o línea de pobreza por encima de la cual todas las necesidades básicas se satisfarían, y en identificar aquellos hogares o personas cuyos ingresos se encuentren debajo de la línea. Siguiendo las definiciones del Instituto Nacional de Estadísticas y Censos (indec), el concepto de Línea de Indigencia determina si un hogar cuenta o no con ingresos suficientes para cubrir una canasta básica de alimentos (СBA), mientras que la Línea de Pobreza (LP) incluye también otros bienes y servicios no alimentarios, tales como vestimenta, transporte, salud, educación, entre otros que configuran una canasta básica total (СBT). 
precios mínimos registrados para los productos integrantes de la canasta excluyendo los centros de compra de gran tamaño (supermercados e hipermercados)" (DNCPM, 2003: 1). La DNCPM partía del argumento de que los precios medios, es decir, los utilizados por el INDEC, no eran representativos de los que pagan los hogares de menores ingresos, porque tienen un alto componente de precios tomados en grandes centros comerciales a donde los pobres (según la DNCPM) no concurren, posiblemente por falta de crédito o por dificultades para acceder a los mismos por razones de distancia.

El INDEC sostuvo que el problema de esta propuesta residía en que resignaba el uso del mejor indicador disponible para medir la evolución de los precios de los artículos de la canasta básica, adoptando alternativas que implicaban una menor calidad en términos estadísticos.

Según el informe del INDEC:

Elegir los precios mínimos (totales o acotados) que son, por lo general, el registro de una sola observación y corresponden a ofertas temporales o valores extremos de una muestra de ocho mil negocios distribuidos en el Gran Buenos Aires implica además, que la canasta valuada a tales precios resultaría absolutamente imposible de adquirir, ya que probablemente los mismos corresponden a observaciones geográficamente dispersas (2003b: 6).

Estas divergencias inciden tanto en la línea de indigencia como en el valor de la línea de pobreza.

El informe del INDEC, lejos de subestimar la importancia de los criterios para valuar los alimentos de la Canasta Básica, enfatizó que la revisión de un aspecto parcial de una metodología estadística, no era el procedimiento más adecuado cuando se cuenta con evidencias que permitirían una actualización integral, como la que estaba siendo llevada adelante por el Instituto.

La otra modificación, propuesta desde Economía, apuntaba hacia los componentes no alimentarios de la línea de pobreza; en otros términos: al cálculo de la inversa del coeficiente de Engel. En este caso, la población de referencia utilizada correspondió al primer quintil de ingreso per cápita. Según la DNCPM, el INDEC consideraba como población de referencia un estrato que tiene "consumos suntuarios", como los que se efectúan en concepto de compra y mantenimiento de vehículos particulares, razón por la cual proponían utilizar como población de referencia al primer quintil de la Encuesta Nacional de Gastos de los Hogares 1996/1997, a fin de contar con una estructura más actualizada del consumo.

Sin embargo, el INDEC argumentó que dicha decisión sería inconsistente, porque no puede tomarse una población distinta a la utilizada para 
valorar la Canasta Básica Alimentaria. La manera correcta consistiría en la utilización de la misma población de referencia, que debe "estar constituida por los hogares ubicados en el umbral inmediato superior a aquellos que apenas satisfacen las necesidades básicas alimentarias". Tomar el primer quintil de ingreso per cápita en lugar del establecido según el criterio metodológico señalado, sirve para estimar el gasto total de esos hogares que "precisamente no satisfacen sus necesidades alimentarias" (2003b: 6). ${ }^{7}$

A sus cuestionamientos de la metodología seguida por la DNCPM, el INDEC añadió que además de utilizar distintas poblaciones de referencia, dicha Dirección recurrió a encuestas realizadas con más de 10 años de diferencia entre una y otra. La justificación por parte de la DNCPM residió en que los coeficientes para estimar los componentes no alimentarios de la Línea de Pobreza, debían contemplar la evolución de los precios relativos de los bienes y servicios ocurrida durante la década de 1990. Sin embargo, la metodología del INDEC ajusta el coeficiente de Engel que se corresponde con la población de referencia del año base, 1985-6, por la evolución de los precios relativos, actualizando el valor de las proporciones implícitas en la relación gasto total/gasto en alimentos original.

El ejercicio metodológico realizado por la DNCPM, implicó que la Línea de Indigencia estimada tuvo en común con la medición del órgano rector del Sistema Estadístico Nacional, la misma población de referencia y la misma composición de la Canasta Básica Alimentaria, cuyas pautas de consumo de alimentos surgen del año base 1985; pero utilizó para valuarla precios más bajos.

Otra diferencia con el método del INDEC estriba en que:

la DNCPM aplica a dicha pauta un componente de consumo no alimentario cuyo registro no se corresponde en el tiempo con el de alimentos, existiendo entre un patrón y otro, 10 años de diferencia y además corresponde al primer quintil de la distribución del ingreso per cápita del hogar. La población de este quintil no alcanza a satisfacer sus necesidades mínimas alimentarias, mucho menos puede suponerse que lo hará con las no alimentarias (INDEC, 2003b: 8).

De lo dicho hasta aquí se deriva que el terreno común que posibilitó esta controversia, estuvo dado por la existencia de una mirada monetarista de

7 Para el área metropolitana, según la Encuesta Nacional de Gastos de los Hogares 1996/1997, los hogares de la población de referencia seleccionados por la dncpm consumen en promedio 2233 kilocalorías diarias por adulto equivalente, es decir, $470 \mathrm{kcal} /$ día por debajo de la dieta mínima, que ya es levemente inferior a la de otros países como Chile, México y Uruguay. 
la pobreza, mirada en la que no se cuestionan las concepciones reduccionistas que subyacen a este tipo de estrategias metodológicas, en las que la pobreza o la indigencia parecieran reducirse a la no posesión de un monto de dinero para acceder a determinados bienes. Al mismo tiempo, estos supuestos compartidos se expresan en la utilización de una misma población de referencia y en la composición de la Canasta Básica Alimentaria. Dicho de otro modo, el análisis de esta controversia permite advertir que la mirada monetarista de la pobreza forma parte del conjunto de presupuestos básicos incuestionables para ambos organismos estatales, por cuanto el foco de la discusión fue la valuación de la canasta.

\section{La dimensión pública de la controversia y su estado público}

Al referirnos a la dimensión pública de las controversias, aludimos, retomando a Dascal (2006), a una particularidad de las mismas, que hace que no puedan ser tratadas como diálogos, sino como cuasi diálogos, en virtud de la participación de una presunta platea que normalmente no interviene como participante, pero que permanece en el fondo de la escena.

Esta característica no debe confundirse con el hecho de que la controversia tome estado público, sino que lo específico de este tipo de confrontaciones es que detrás de cada exigencia pragmática habitual, manifestada por un oponente, existe otra exigencia, presumiblemente del mismo tipo, debida a una platea, de modo que las reacciones de los protagonistas de las controversias obedecen a ambos factores (Dascal, 2006). Hablar de la dimensión pública de las controversias, no es sólo reconocer que las mismas puedan tomar estado público; implica asimismo el reconocimiento de que la trama argumentativa que construyen los contrincantes, no sólo tiene en cuenta los argumentos esgrimidos por el o los oponentes, pues en ella se despliegan estrategias tendientes a captar el beneplácito de una presunta platea.

Habiendo hecho esta distinción, es posible afirmar que cuando la discusión tomó estado público, comenzó a reproducirse añadiendo hilos al tejido de la matriz en que se desarrollan estas polémicas. En este contexto, aumentó el número de actores que se pronunciaron respecto de la validez o inexactitid de las mediciones de la pobreza realizadas por el INDEC desde la cartera de Economía. ${ }^{8}$

8 Cabe recodar que el indec es un organismo descentralizado del Estado que responde a la Secretaría de Política Económica, que a su vez tiene como superior directo al ministro de Economía. 
Diversos sectores comenzaron a criticar los cálculos realizados por la DNCPM. ${ }^{9}$ Entre esas críticas destacan la de Claudio Lozano (Central de Trabajadores Argentinos) ${ }^{10}$ y la de Abraham L. Gak, director del Plan Fénix de la Universidad de Buenos Aires. Éste afirmó que ciertas prácticas de poder no cambian con los cambios de gobierno, y que una de estas prácticas es la tentación de presionar al Instituto Nacional de Estadísticas y Censos para que manipule su información. ${ }^{11}$

[...] en la era de la pobreza masiva, el actual ministro de Economía cuestiona el trabajo del INDEC y anuncia sus propias estimaciones de pobreza, cuestionando la validez de las conclusiones alcanzadas por el Instituto. Esto sienta un precedente potencialmente grave. Además, un accionar de este cariz termina siendo un boomerang, al afectar la credibilidad del propio gobierno [...] una vez que se empieza a recorrer el camino de la manipulación de las estadísticas se corre el riesgo del que no hay retorno. La corrección de las cifras relativas a la incidencia de la pobreza del INDEC se funda sobre un procedimiento sin sustento técnico (página 12, 31 de agosto de 2004).

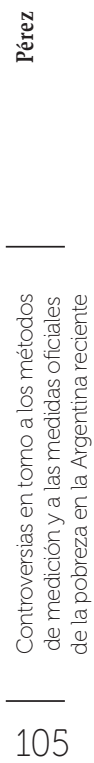

A mediados de 2005, el ex ministro de economía se opuso nuevamente a las cifras arrojadas por el INDEC, esta vez apoyando sus argumentos en datos proporcionados por el Banco Mundial, ${ }^{12}$ poniendo en un segundo plano o eludiendo el foco original de la controversia - que se centraba en lo metodológico - , y reenviando esta controversia al contexto de las discusiones que involucran a los organismos internacionales y a los institutos de estadísticas públicas, ${ }^{13}$ lo cual puso en evidencia la motivación propa-

Durante esta primera etapa, el Ministerio de Economía estuvo a cargo del Lic. Roberto Lavagna, y la dirección del indec estuvo en manos del Lic. Juan Carlos del Bello hasta la asunción de Néstor Kirchner a fines de mayo de 2005, que designó en este lugar al Lic. Lelio Mármora.

9 "Las cifras de Lavagna no cayeron en saco roto. Al día de ayer eran diversas las críticas que señalaban desconfianza hacia la metodología utilizada por el Ministerio de Economía para calcular una rápida reducción de la pobreza (del 55 al 46 por ciento entre octubre de 2002 y marzo pasados) y la indigencia (del 26 al 16 por ciento en el mismo período)" (Página 12, 29 de julio de 2004).

10 "[Lavagna] busca bajar el número de pobres por vía de una alquimia estadística de dudosa seriedad, con el objetivo de ocultar la pobreza" (Página 12, 29 de julio de 2004).

11 En el mismo artículo, Gak recuerda que el ex ministro de economía, Domingo Cavallo, solía cuestionar los índices de desempleo, tanto en su gestión de la década del 90 como en la que concluyó con la crisis de diciembre de 2001.

12 "a contramano de los datos que difundió el indec la semana pasada, el ministro de Economía, Roberto Lavagna, tomó datos de Banco Mundial y afirmó que se rompió la tendencia al aumento de la brecha entre ricos y pobres 'por primera vez en 11 años' " (Clarin, 5 de julio de 2005).

13 Es importante aclarar que al reconstruir los modos en que fueron difundidas estas controversias, es posible considerar a la prensa escrita un actor dentro de esta matriz, y no como mera 
gandística implícita en el sostenimiento de una controversia que se planteó originalmente como metodológica; es decir, el ex ministro jugó el juego de las controversias de cara a una supuesta platea.

Así pues, aunque la controversia original tuvo como foco cuestiones metodológicas ligadas a los criterios divergentes que, según dos dependencias del Estado, deben emplearse para medir la pobreza, a partir de la difusión de los datos que contradecían a los publicados por el organismo rector del Sistema Estadístico Nacional, se puso de manifiesto la extensa matriz de actores involucrados en dicha polémica.

En línea con los anterior, las controversias que se dieron entre 2003 y fines de 2005 en torno a los métodos y mediciones que, como una alternativa a los del INDEC, impulsó la Dirección Nacional de Políticas Macroeconómicas, constituyeron un antecedente tanto de los problemas como de las prácticas que iniciaron un proceso de debilitamiento de la confianza en las estadísticas públicas que trasciende el estricto dominio de la medición de la pobreza.

Así las cosas, a lo largo de 2006 identificamos otra etapa en la que el INDEC siguió siendo protagonista de controversias, encabezadas ahora por otro organismo del Estado: la Secretaría de Comercio Interior.

El eje de estas controversias no se circunscribió a las mediciones de pobreza, sino que incluyó discusiones metodológicas respecto a la manera de relevar el Índice de Precios al Consumidor, ${ }^{14}$ insumo indispensable para

reproductora de los discursos de otros contendientes más visibles (indec-dncpm), pues no se trata de actores imparciales en lo referente al modo en que presentan las posiciones de los protagonistas de las controversias, ni respecto a la forma en que construyen su relato del vínculo entre mediciones y pobreza. Clarín, La Nación y Ámbito Financiero en general se limitan a reproducir datos numéricos respecto de la pobreza, sin esclarecer en qué sentido, desde qué conceptos o métodos, o bajo qué presupuestos están construyendo su discurso respecto de la pobreza, contribuyendo de esa manera a un vacío conceptual que impide comprender las condiciones bajo las que se realiza la medición, qué se está midiendo al realizar dichas mediciones o qué se está discutiendo. De este modo, el problema de la pobreza queda reducido a una disputa más centrada en la cifra que en el método; a una disputa política, en la que se diluye el sentido de lo que originalmente se estaba discutiendo. Así como los gobiernos pueden utilizar las estadísticas públicas como propaganda, también los medios pueden hacer uso de ellas en sentido inverso, deslegitimando las cifras o haciendo uso de las mismas para brindar apoyo a los presupuestos ideológicos a partir de los cuales construyen sus propios discursos tanto de las gestiones como de la pobreza.

14 Indudablemente, gran parte de las discusiones que se dan respecto del IPC tienen como telón de fondo la sanción de la ley 25713, en cuyo primer artículo se establece que "A las obligaciones que en origen hubieran sido expresadas en dólares estadounidenses u otra moneda extranjera y que hubieren sido transformadas en pesos a partir de la sanción de la ley 25.561 o bien posteriormente, se les aplicará un Coeficiente de Estabilización de Referencia (CER) que se compondrá por la tasa de variación diaria obtenido de la evolución mensual del Índice de Precios al Consumidor (IPC) publicado por el Instituto Nacional de Estadística y Censos dependiente del Ministerio de Economía de la Nación" (dicha ley fue sancionada el 28 de diciembre de 2002 y 
el cálculo de las Líneas de Pobreza e Indigencia, así como el ejercicio de ciertas prácticas que derivaron en procesos judiciales.

Si bien las polémicas entre la Secretaría de Comercio Interior y el área del IPC del INDEC, se encuentran vinculadas a la que es eje de nuestro análisis, el desarrollo de las mismas excede las pretensiones de este trabajo, motivo por el cual no profundizaremos en ellas; nos limitaremos a señalar que la relación entre una y otro comenzó a resquebrajarse ante sucesivos pedidos de información que desde dicha Secretaría se hacían al Instituto, excediendo los niveles de desagregación previstos en la metodología 13, vigente desde 1999. Esto motivó que el entonces director del Instituto convocara a la "Comisión de Consulta sobre Secreto Estadístico", a fin de tomar una decisión respecto a qué información podía brindarse. Esta comisión emitió un dictamen en julio de 2006, fijando el alcance de la información que podía entregarse sin violar dicho precepto. ${ }^{15}$

En una carta enviada al secretario de Política Económica, el entonces director del INDEC había expresado:

[...] durante los últimos meses ha habido una marcada diferencia entre el uso cuidadoso de una información pública, a la cual tienen acceso los decisores políticos para hacer más eficiente su gestión, y lo que estamos observando en su actual utilización.

promulgada parcialmente el 8 de enero de 2003). A partir de esto, existen interpretaciones que apuntan a que la manipulación de los datos del IPC está orientada a disminuir el valor de los bonos de deuda pública.

Según establece el artículo décimo de dicha ley, las informaciones que suministran los organismos que integran el Sistema Estadístico Nacional, son estrictamente secretas y sólo pueden utilizarse con fines estadísticos (Artículo 10, ley 17622/68). Asimismo, el artículo 13 señala que todas las personas que por razón de sus cargos o funciones tomen conocimientos de datos estadísticos o censales, se encuentran obligados a guardar sobre ellos absoluta reserva. En línea con lo anterior, el artículo 14 establece que las personas que deban realizar tareas estadísticas o censales, con carácter de carga pública, estarán obligadas a cumplir estas funciones. Si no lo hicieran, se harán pasibles de las penalidades preceptuadas en el artículo 239 del Código Penal, salvo que aquéllas estuviesen comprendidas en las excepciones que establezca reglamentariamente el Poder Ejecutivo. El artículo 15 establece que quienes no suministren en término falseen o produzcan con omisión maliciosa las informaciones necesarias para las estadísticas y los censos, estarán incurriendo en una infracción pasible de ser multada. Finalmente, el artículo 17 establece que: “Los funcionarios o empleados que revelen a terceros o utilicen en provecho propio cualquier información individual de carácter estadístico o censal, de la cual tenga conocimiento por sus funciones, o que incurran dolosamente en tergiversación, omisión o adulteración de datos de los censos o estadísticas, serán pasibles de exoneración y sufrirán además las sanciones que correspondan conforme con lo previsto por el Código Penal (Libro II, Título V, Capítulo III) a cargo del Sistema Estadístico Nacional.". Ley 17622/68. 
[...] estamos preocupados frente al peligro de perder la credibilidad en el IPC, lo cual podría afectar la información oficial con relación a un indicador tan sensible, así como la credibilidad de los otros indicadores que produce el INDEC (FIA, 2007a: 33). ${ }^{16}$

\section{De las controversias al conflicto}

A diferencia de la etapa 2003-2005, en la cual la controversia entre la Dirección Nacional de Coordinación de Políticas Macroeconómicas y el Instituto Nacional de Estadísticas y Censos, respecto a mediciones y metodologías alternativas a las del INDEC, adquirió estado público, en 2006 la controversia se invisibilizó, hubo un repliegue del estado público de la misma, lo cual no implica que se hayan resuelto las tensiones ni que aun en una etapa de invisibilización carezcan estos cuasi diálogos (Dascal, 2006) de una dimensión pública. Justamente, la característica de estos intercambios tiene que ver con que los contrincantes adoptan estrategias pensando en una presunta platea.

Las discusiones respecto al Índice de Precios al Consumidor y de los restantes índices que lo utilizan como insumo, se transformaron a mediados de enero de 2007, inaugurando la que consideramos tercera etapa dentro del periodo estudiado. El hito que marcó este "punto de inflexión en la historia del sistema estadístico argentino" (Otero, 2011), fue la intervención del organismo, expresada en la incorporación de la Lic. Beatriz Paglieri, a solicitud de la entonces ministra de Economía, Felisa Miceli, a la comisión del INDEC - que se conformó con el objetivo de analizar los problemas que estaban surgiendo en la medición del IPC - , y en su vertiginoso ascenso a directora del IPC.

En el marco de su gestión, y según se desprende de la investigación realizada por la Fiscalía de Investigaciones Administrativas de Nación, los datos correspondientes al IPC de enero fueron adulterados, provocando una situación que ha llegado a ser definida en el ámbito académico como de "manipulación impúdica" (Torrado, 2007).

En este contexto, en febrero de 2007 un grupo de senadores opositores solicitaron a la Fiscalía de Investigaciones Administrativas de la nación, el inicio de una investigación que permitiera determinar la existencia de presuntas irregularidades administrativas y/o comisión de delitos que habrían sido realizados por el secretario de Comercio Interior, pues tal como reseñamos anteriormente, dicho funcionario habría solicitado información

16 Anexo de la investigación realizada por la Fiscalía de Investigaciones Administrativas (2007a: 33). 
amparada en la ley de secreto estadístico, en vigencia desde la creación del propio INDEC. Seguidamente, entre febrero y marzo de 2007, renunció la directora nacional de Estadísticas de Condiciones de Vida. ${ }^{17}$

A partir de esto, diversos sectores comenzaron a denunciar la intervención del organismo, e ingresaron en escena distintos actores, a saber: empleados del instituto, sindicatos, la comunidad científica de profesionales de ciencias sociales y económicas, y ex directores del instituto, entre otros. ${ }^{18}$

En el plano internacional, los coordinadores regionales y nacionales del Programa de Comparación Internacional (PCI) en América del Sur, manifestaron su

alarma y preocupación por la decisión tomada por el ministro responsable del INDEC de reemplazar a la directora del área, por alguien sin la experiencia ni los conocimientos necesarios. El reemplazo de la funcionaria, coincide también con la introducción de cambios metodológicos en el IPC que no están basados en criterios estadísticos o económicos establecidos internacionalmente sino en función de necesidades ajenas a los objetivos técnicos del IPC. Esto genera desconfianza adicional y daña la credibilidad no solamente del IPC argentino sino también de la integridad profesional de los miembros de la comunidad estadística internacional. ${ }^{19}$

Sumándola a las expresiones públicas ya existentes de repudio a las prácticas que estaban produciéndose dentro del instituto por parte de profesionales de diversas instituciones de corte académico, los trabajadores del INDEC iniciaron una serie de acciones tendientes a ampliar el público de las controversias. Así, a la puerta del Instituto y en un escenario que

17 La licenciada Clyde Charre de Trabuchi, graduada en Estadística por la Universidad Nacional de Rosario y ex directora nacional de Estadísticas de Condiciones de Vida, asumió en 2008 la presidencia del Instituto Interamericano de Estadística (IASI), entidad en la que se concentran todas las dependencias de estadística de los países de América, y las principales personalidades profesionales, académicas y científicas de la disciplina.

18 La protesta de un grupo de trabajadores del INDEC frente a la inminente publicación de los datos del Índice de Precios al Consumidor nacional en el mes de febrero, reenvió a la esfera pública el conflicto que se estaba desarrollando en el Instituto. Simultáneamente, se dio a conocer una solicitada en diversos medios gráficos, que fue también reproducida en distintos artículos periodísticos y a la cual era posible acceder en la web firmada por más de 200 profesionales de ciencias sociales, económicas y estadísticas. Dicha declaración apuntaba a expresar públicamente la preocupación y alarma que habían generado en la comunidad científica el desplazamiento de la funcionaria que estaba a cargo de la Dirección de Índices de Precios al Consumo y los alcances que dicha medida tenían en cuanto a "la calidad, continuidad y transparencia" de un indicador tan significativo para la población y las propias autoridades gubernamentales.

Disponible en http:/ /indecdepie-adhesiones.blogspot.com/ 
contaba con la presencia de policías uniformados, técnicos y encuestadores del organismo denunciaron ante diversos medios las presiones a los trabajadores y las modificaciones en el manejo de las encuestas del área del IPC.

En el mes de marzo se realizó una audiencia pública en el Congreso de la Nación. Allí la presidenta de la Sociedad Argentina de Estadística (SAE) sostuvo que la tarea fundamental para la creación del "dato" corresponde al "estadístico", lo cual implicaba que no se trata de una tarea en la que deban involucrarse funcionarios políticos, y luego agregó que:

Se debe contar con un INDEC que aplique metodología estadística apropiada, que los profesionales sean idóneos en la disciplina y que se trabaje con ética, lo cual beneficia no sólo a los funcionarios de la institución, sino también al poder político, a las instituciones de formación académica y al país en general, porque como es ampliamente sabido, contar con información cuantitativa confiable y a tiempo, es primordial para poder construir políticas y tomar decisiones acertadas. ${ }^{20}$

En este contexto, el director del INDEC presentó su renuncia. ${ }^{21}$ Meses más tarde se presentó al Ministerio de Economía el Programa Estadístico Nacional 2007-2011. Dicho plan contemplaba un proceso de transformaciones en el que resultaba "necesario proceder al cambio de algunos directores". Los reemplazantes serían, según consta en el documento Lineamientos del Programa Estadístico Nacional, personal de carrera del instituto. ${ }^{22}$ Esta decisión, planteada al presentarse el proyecto, sólo contribuyó a deslegitimar las propuestas planteadas, pues los procesos de transformación o introducción de nuevas metodologías, suelen requerir la construcción de un consenso brindado por la comunidad científica y técnica involucrada en el tema, más que del desplazamiento y silenciamiento de actores con experiencia que podrían no sólo aportar su conocimiento para enriquecer las discusiones, sino también legitimar los patrones metodológicos del Instituto.

Entre las pautas del programa, destacaba la convocatoria a asesoramiento internacional, con miras a implementar "mejores prácticas" que aseguraran la calidad de los procesos de producción de estadísticas y la

\footnotetext{
20 La desgravación de la exposición se encuentra completa en: http://indecdepie.blogspot. com/2007/06/clase-pblica-en-el-congreso-de-la-nacin_27.html

21 El Lic. Mario Krieguer ocupó transitoriamente el cargo dejado por el Lic. Lelio Mármora, y luego fue reemplazado por el Lic. Alejandro Barrios.

22 "Lineamientos del Programa Estadístico Nacional", Instituto Nacional de Estadísticas y Censo, 2007, p. 5.
} 
comparabilidad en el plano internacional, lo cual evidencia una cierta relación de dominación implícita en la adopción de sistemas clasificatorios o métodos que, como manifiesta Torrado (2007), se presentan como "canonizados" por contar con el sello de "recomendación internacional".

Hasta aquí, la falta de credibilidad respecto de los cálculos del IPC parecía no afectar más que tangencialmente las estadísticas de pobreza; sin embargo, puesto que el IPC es indispensable para el cálculo de las líneas de pobreza e indigencia, consideramos importante reseñar someramente estas polémicas interrelacionadas, mostrado a los diversos actores involucrados, los argumentos y las acusaciones, pues todo ello forma parte del espacio controversial (Nudler, 2009) que es objeto de nuestro análisis.

\section{Conflictos en torno a la medición de la pobreza}

La vinculación Índice de Precios al Consumidor -Línea de Pobreza, se puso expresamente de manifiesto y cobró estado público, a partir de la negativa de la directora de la Encuesta Permanente de Hogares, Lic. Cynthia Pok, a realizar el cálculo de pobreza e indigencia correspondiente al primer semestre de 2007, debido a la falta de datos confiables.

Los cambios introducidos en la elaboración del Índice de Precios al Consumidor (IPC) del INDEC desde enero de 2007 a la fecha, ponen en cuestionamiento el uso del IPC actual como insumo para el cálculo de la CBA y la CBT que debería utilizarse para la medición de la pobreza en el primer semestre de 2007. Las razones técnicas que invalidan su uso ya fueron expuestas en ocasión de la no elaboración de los informes de prensa referidos a la "valorización mensual de la Canasta Básica Alimentaria y de la Canasta Básica Total correspondientes a los meses de enero a junio de 2007.23

Tal como se desprende de la cita anterior, el eje de la discusión se puso en argumentos metodológicos o técnicos; Pok destacó que al introducirse modificaciones en un indicador, es indispensable presentar asimismo la magnitud de la incidencia de dichos cambios sobre la serie que se venía calculando; e hizo hincapié en que la presentación de esta incidencia es la herramienta metodológica que permite distinguir los cambios debidos a

23 Memo interno, 2 de julio de 2007, enviado al entonces director del indec Alejandro Barrios, con copia a la Lic.Edwin y refrendado por 16 trabajadores con distinto nivel de responsabilidad en el cálculo de los índices de pobreza. 
la evolución de los precios, de los cambios producidos por el efecto metodológico. "Sin esa articulación no es posible dar cuenta del empalme de la serie, siendo ésta una normativa básica de la práctica metodológica en la producción de estadística pública." Finalmente, y en virtud de lo expuesto, sostuvo que no se contaba con las condiciones necesarias "para la elaboración de los indicadores de pobreza e indigencia del primer semestre de 2007 con las características de rigurosidad técnica con que siempre se ha trabajado".

Las razones expuestas por Pok no fueron respondidas en el marco de una discusión metodológica; en lugar de generar argumentos alternativos, es decir, una genuina controversia, la respuesta se salió de los cauces que podían conducir al entendimiento, y ella fue desplazada de su cargo, ${ }^{24}$ deslegitimándola como interlocutora válida, requisito indispensable para que existan controversias. La ausencia de una mínima bilateralidad, propia de una controversia, puso de manifiesto que se había ingresado en el terreno del conflicto.

La remoción de la directora de la Encuesta Permanente de Hogares, motivó una serie de declaraciones públicas por parte de distintos actores, ${ }^{25}$ lo que muestra parte del contexto en que se generan los conceptos y las estrategias de medición de la pobreza. Trabajadores del INDEC, fundamentalmente los ligados a la Encuesta Permanente de Hogares, se solidarizaron con ella e iniciaron un paro activo por una semana. Investigadores, docentes, personal de apoyo y personal técnico de diversas instituciones del sistema científico y tecnológico, expresaron su preocupación, tanto por los hechos que venían ocurriendo desde enero de 2007 como por el desplazamiento de la Lic. Pok, ya que estos acontecimientos, según manifestaron, no sólo ponían en duda la calidad de los datos elaborados, sino que también ignoraban el alto nivel profesional del personal, generando sospechas acerca de los motivos que sustentaban las decisiones institucionales. ${ }^{26}$ En el mismo sentido se expresó un grupo de trabajadores del Ministerio del Trabajo, la Sociedad Argentina de Estadística (SAE), la Asociación Argentina de Especialistas en Estudios del Trabajo (ASET), la Asociación de Estudios de Población de Argentina (AEPA) ${ }^{27}$ y el Instituto Interamericano de Estadística. ${ }^{28}$

\footnotetext{
24 Decreto 870/07

25 http://linksarg.com.ar/cuidemosalINDEC/

26 http://indecdepie-adhesiones.blogspot.com/

27 http://indecdepie-adhesiones.blogspot.com/

28 Boletín informativo del Instituto Interamericano de Estadística, núm. 73, junio 2007.
} 
Meses más tarde, fueron cesados varios trabajadores ligados al área de precios y de la encuesta permanente de hogares. ${ }^{29}$ Las expresiones de repudio desde otras instituciones continuaron. El Consejo Directivo de la Facultad de Ciencias Exactas y Naturales de la Universidad de Buenos Aires, también se pronunció respecto al conflicto del INDEC. ${ }^{30}$ La junta de la carrera de sociología de la UBA y del Colegio de Sociólogos de la provincia de Buenos Aires, apoyaron el abrazo al INDEC realizado por un sector de los trabajadores del Instituto a principios de diciembre de 2007. ${ }^{31}$

A fines de enero de 2008, un grupo de trabajadores del INDEC enrolado mayoritariamente en la Asociación de Trabajadores del Estado (ATE), publicó sus propias estimaciones de inflación, cuestionando una vez más la validez de los datos oficiales. Frente a esto, la directora del organismo respondió con un comunicado interno que fue distribuido entre el personal del Instituto y donde afirmaba que la dirección del mismo se encontraba legítimamente instituida a través del Decreto 1076/07.

La Dirección del INDEC, legítimamente instituida, reafirma que la información producida por este instituto resulta absolutamente confiable y se ajusta a los estándares de calidad internacionales, a la vez que rechaza por falsa la irresponsable denuncia sobre "manipulación de datos".

A modo de conclusión, quienes se presentan públicamente como "técnicos del INDEC", detrás de una supuesta impugnación metodológica esconden una trama de defensa de privilegios, ocultamiento de maniobras realizadas con anterioridad para beneficio de diversos actores políticos y sociales, así como también vienen a pretender legitimar los resultados informados por esas consultoras que no gozan ni de la trayectoria, ni de la estructura, ni de la experiencia que acreditan esta institución y sus empleados.

29 Según declaraciones de la Junta Interna de la Asociación de Trabajadores del Estado, la mayoría de los desplazados habían prestado declaración en la fiscalía en la causa abierta por "violación del secreto estadístico".

30 Dirigiéndose al presidente de la nación y al ministro de Economía, manifestaron la preocupación del Consejo Directivo de dicha casa de estudios por la situación del Instituto, destacando la necesidad de que el mismo desarrolle sus actividades en un marco en el cual esté garantizada la independencia y la excelencia técnica. Luego instaron a respetar el acta acuerdo firmada el 17 de octubre de 2007, reincorporando a los trabajadores despedidos, y avanzando en la recuperación del indec, transparentándolo y despojándolo de manipulaciones políticas. Finalmente, expresaron su solidaridad con los trabajadores despedidos y se adhirieron a las jornadas de lucha contra la intervención política de dicho organismo. (Memo, reunión Consejo Directivo, 5 de noviembre de 2007.)

31 La adhesión está fechada el 20-11-07 
Por último, esta Dirección afirma que en el organismo no se han producido despidos ni se han llevado a cabo acciones de hostigamiento. La reformulación de procesos de trabajo y las decisiones de actualización metodológica son responsabilidad primaria de los niveles de conducción del organismo, conforme a la normativa vigente. ${ }^{32}$

Si bien ya desde enero del 2007 se había ingresado en el terreno del conflicto y fue clausurada la posibilidad de mantener controversias en cuanto debate racional, por la anulación del otro como interlocutor válido, creemos que las estrategias para sostener el conflicto en este comunicado, manifiestan parte de los artilugios desplegados por los contendientes de las controversias que describe Dascal (2006), ya que la réplica a los cuestionamientos metodológicos se dirige hacia acusaciones de otro orden, intentando generar sospechas en un supuesto público y tratando de deslegitimar dicha acusación. La legitimidad de sus métodos y mediciones, pareciera derivar de que es un Instituto con un tipo de dominación legal con administración burocrática. Pero la legitimidad de los instrumentos y productos de la estadística pública no se desprende linealmente de la legalidad de los nombramientos ni de la adopción de estándares internacionales, sin mencionar, definir o explicitar cuáles son esos estándares, como si los mismos fueran intrínsecamente objetivos, válidos y confiables por el hecho de no ser nacionales. La trayectoria, la estructura y las experiencias acreditadas por el INDEC, son presentadas como recursos apropiables por quienes ocupan cargos ejecutivos, y no como fruto de procesos complejos y largos de construcción de confianza respecto a la utilidad y confiabilidad de la estadística pública, las cuales deben revalidarse a través de prácticas.

\section{De las controversias sobre los métodos de medición a las discusiones respecto a las mediciones de la pobreza}

A lo largo del proceso controversial analizado, identificamos un primer periodo caracterizado por controversias cuyo foco se centraba en lo metodológico, es decir, en distintas visiones respecto a los instrumentos de medición; esta situación se modifica a partir de 2008, cuando las discusiones pasan a tener como foco, no el proceso de construcción del instrumento, sino los resultados del mismo.

32 Comunicado interno, firmado por la Dirección del Instituto y dirigido al personal del INDEC el 30 de enero de 2008. 
Esta controversia, si bien podría retomar, en cierto modo, una preocupación común respecto de las estadísticas, es decir, respecto al grado de verdad de éstas, a su capacidad de reflejar u ocultar, mediante la precisión del número, los procesos sociales estudiados, se planteó más bien en términos políticos, lo que puso de manifiesto la coexistencia de los diversos lenguajes - el administrativo, el político y el sociológico -, no exentos de tensiones, que caracterizan la producción de la estadística pública.

Así, en mayo de 2008, luego de un año en que prácticamente no publicó estimaciones de pobreza, el INDEC dio a conocer, a través de una escueta nota de prensa, una tendencia decreciente en los porcentajes de hogares y personas situados por debajo de las líneas de pobreza e indigencia en el periodo comprendido entre el último trimestre de 2007 y el primero de 2008. ${ }^{33}$

Frente a la publicación de estos datos, hubo distintas reacciones ${ }^{34}$ que dan cuenta de la imprevisibilidad de las controversias (Nudler, 2009), es decir, de la probabilidad de que se extiendan hacia temas insospechados, o de que aparezcan actores no contemplados en el origen de las mismas. Esta característica se puso en evidencia cuando los jerarcas de la Iglesia católica $^{35}$ afirmaron categóricamente que la pobreza estaba aumentando en Argentina.

En línea con lo anterior, el documento Hacia un bicentenario en justicia y solidaridad 2010-2016, producto de la $96^{\text {a }}$ asamblea plenaria de obispos, manifestaba que los argentinos cuentan con la capacidad de erradicar la pobreza, distinguiéndose de quienes (aunque no son nombrados explícitamente, asumimos que se trata del gobierno) reducen el problema a un tema meramente estadístico. "Creemos que existe la capacidad para proyectar, como prioridad nacional, la erradicación de la pobreza y el desarrollo in-

33 Según explica el comunicado del indec, la conformación particular de este semestre obedeció a que en el tercer trimestre de 2007 no se relevaron los aglomerados Gran Buenos Aires, Mar del Plata-Batán, Bahía Blanca-Cerri y Gran La Plata. La falta de datos de ese periodo habría obedecido al paro realizado por los trabajadores del Instituto ante los hechos descritos previamente. Entre las mismas cabe destacar la de la ex directora de la Encuesta Permanente de Hogares del indec, quien sostuvo que desde que el equipo responsable de la encuesta permanente de hogares se negó a calcular la pobreza por las sospechas de manipulación del ipc, "la intervención del indec armó un equipo ad hoc para calcularla. Nadie sabe cómo calcularon la cifra de pobreza, pero no fue el equipo técnico de la Encuesta Permanente de Hogares". Según Pok, el equipo de la EPH siguió "haciendo la encuesta de hogares y procesando la información". Pero sin hacer "el cálculo porque no contamos con una medición de los precios de la canasta". Además, señaló que "las bases de datos de los ingresos de las personas que se relevan en todo el país son enviadas con copia a la intervención del organismo", pero que no se sabe qué precios utilizan las actuales autoridades del Instituto para medir la pobreza (Crítica, 13 de mayo de 2008).

35 Al hablar de iglesia católica nos referimos a su alta jerarquía, sin ignorar que dentro del catolicismo existen muy diversos actores, con concepciones y prácticas también variados. 
tegral de todos. (...) No se trata solamente de un problema económico o estadístico." 36

La alta jerarquía eclesiástica se considera parte de la política argentina, y es desde esta autopercepción que juzga legítimo pronunciarse respecto de cuestiones como las mediciones de la pobreza. Su denuncia respecto a la manipulación de estadísticas, se vincula no sólo con la autopercepción mencionada, sino también con su afición a imponer los modos de organización familiar y las prácticas sexuales (Esquivel, 2000). En un documento titulado "El gobierno y la manipulación de la información para la despenalización del aborto", la cúpula de la Iglesia sostiene la existencia de "un manejo desleal y malicioso" de datos por parte del gobierno, y lo acusan de manipular la opinión pública a través de "activistas" pertenecientes a distintas organizaciones sociales que trabajan a favor de "caprichos ministeriales". ${ }^{37}$

Según la Agencia Informativa Católica, la Asociación Civil Portal de Belén denunció ante la Justicia Federal de Córdoba, en 2005, al entonces ministro de Salud de la Nación, por difundir "estadísticas falsas de los abortos que se practican en la Argentina" a través de los medios masivos de comunicación.

Por cuanto son clara expresión de una de las vertientes del catolicismo antiliberal, para los denunciantes "el actual gobierno nacional cumple los deseos inconfesables de los organismos multilaterales de crédito - los únicos acreedores que cobran por adelantado - , y de las agencias de la ONU, cuyo programa es: 'eliminemos a los pobres, para eliminar la pobreza'". ${ }^{38}$

En el mismo sentido, la problemática de la pobreza y la tergiversación de datos aparece en documentos eclesiásticos, como el emitido por el Secretariado Nacional para la Familia, en el cual - tras haber analizado, dice, la "Propuesta de Protocolo para la Atención Sanitaria de casos de Abortos Legales", publicada por el Instituto Nacional contra la Discriminación, la Xenofobia y el Racismo - se sostiene que "para los organismos del Estado, la pobreza se combate con el aborto". Y este informe del Secretariado agrega que quizás haya llegado el momento de "poner freno al 'desenfreno abortista'. Todos sus argumentos son rebatibles desde la ciencia, la economía y la sociología" (Buenos Aires, 12 de septiembre de 2007). ${ }^{39}$

\footnotetext{
36 Los obispos de la Argentina, 96² Asamblea Plenaria. El Cenáculo-la Montonera (Pilar), 14 de noviembre de 2008, "Hacia un bicentenario en justicia y solidaridad 2010-2016".

39 Para comprender el contexto en el que se dieron estas discusiones, es importante aclarar que el gobierno de Kirchner se ha mantenido distante del poder episcopal y del Vaticano, y activo con grupos católicos y religiosos. El abandono de la designación de ilustres católicos que devenían
} 
Así, desde distintos sectores de la Iglesia, por diversas vías e intentando amparar sus dichos en disciplinas científicas, aunque sin especificar el modo en que desde las mismas se rebatirían estos enunciados, se acusa a los organismos del Estado lo mismo de subestimar las cifras de la pobreza que de sobreestimar la correlación pobreza-aborto-muerte materna. Estos pronunciamientos evidencian que la Iglesia, en tanto institución claramente opositora, se disputa con el Estado, y con este gobierno en particular, no sólo el poder de decir, nombrar o definir quiénes son pobres, sino también el poder de contarlos. ${ }^{40}$

Finalmente, dentro del periodo iniciado en 2007, es posible identificar una nueva fase a partir de la implementación, a fines de 2009, de un programa de transferencia condicionada de ingresos conocido como "Asignación Universal por Hijo", ${ }^{41}$ que disminuyó los niveles de pobreza por ingreso, ${ }^{42}$ concepto que, como sostuvimos anteriormente, aparecía como incuestionable. El escenario configurado a partir de la implementación de esta política con alto grado de universalidad, sin duda debilita el conflicto acerca de las mediciones, motivo por el cual se evidencia una disipación de las controversias en torno a los resultados de las mismas.

funcionarios públicos en nombre del poder eclesiástico y su reemplazo por funcionarios de un gobierno que intenta mostrar autonomía respecto de los poderes establecidos, así como el no alineamiento del Estado argentino con el Estado Vaticano en los encuentros de Naciones Unidas referidos a temas de sexualidad y mujer, o la postura del gobierno respecto a la dictadura que vivió Argentina, sin duda brindan coordenadas para comprender las posturas de los distintos actores que intervienen en este conflicto (Mallimaci, 2005). Informe técnico $N^{\circ}$ 69, Secretariado Nacional para la Familia (SNPF), Buenos Aires, 12 de septiembre de 2007.

41 Mediante el decreto del P.E.N 1602/09 del 30 de octubre de 2009, se creó la Asignación Universal por Hijo para la Protección Social (AUH), una política que brinda una prestación no contributiva similar a la que reciben los hijos de los trabajadores formales, pero en este caso dirigida a "aquellos niños, niñas y adolescentes residentes en la República Argentina, que no tengan otra asignación familiar prevista por la presente ley y pertenezcan a grupos familiares que se encuentren desocupados o se desempeñen en la economía informal". Dicha política no sólo buscaba mejorar los indicadores de bienestar social, sino también solucionar uno de los problemas del nuevo modelo macroeconómico dado por el hecho de que si bien muchos sectores han mejorado su calidad de vida, ciertos grupos históricamente vulnerables (niños, madres solas y familias numerosas, entre otros) habían aumentado su fragilidad en los últimos años. Cabe destacar que el cobro de la AUH es incompatible con la percepción de otros planes sociales y excluye del beneficio a aquellos trabajadores del sector informal que perciban ingresos mensuales superiores al Salario Mínimo Vital y Móvil vigente. El 20\% del estipendio previsto en la AUH se cobra acumulado a principios de cada año lectivo contra la presentación de los certificados de escolaridad y vacunación.

42 Véase Agis, E. Cañete, C. y D. Panigo, 2011, El impacto de la Asignación Universal por Hijo en Argentina, CEIL-PIETTE CONICET, documento de trabajo; E. Roca, 2011, “La Asignación Universal por Hijo. Extensión de las asignaciones familiares" pp. 30-43. 


\section{A modo de cierre}

La polémica que la Dirección Nacional de Coordinación de Políticas Macroeconómicas entabló, entre 2003 y 2005, con el Instituto Nacional de Estadísticas y Censos, respecto de cuestiones metodológicas ligadas a la medición de la pobreza, a nuestro entender constituyó el inicio de un proceso cuyo desenlace difícilmente hubiésemos podido proyectar en aquel entonces. En este primer periodo, el terreno común que posibilitó las controversias estuvo dado por la existencia de una mirada monetarista de la pobreza, impuesta y avalada por organismos internacionales como el Banco Mundial; en dicha mirada, las concepciones reduccionistas que subyacen a este tipo de estrategias metodológicas, se presentaron como incuestionables para ambas dependencias estatales, y el núcleo de las distintas posiciones fue la valuación de la canasta básica y las poblaciones de referencia.

En 2006 se produjo un repliegue de dicha disputa, un corrimiento del eje de las discusiones hacia el Índice de Precios al Consumidor (insumo indispensable para el cálculo de las Líneas de Pobreza e Indigencia), por lo que hicieron su aparición nuevos actores, enrolados en el Instituto Nacional de Estadísticas y Censos, y en la Secretaría de Comercio Interior. Esta controversia, a nuestro entender, fue clausurada a principios de 2007.

La suspensión de la posibilidad de sostener controversias, lejos de implicar una resolución o el abandono, derivó en un conflicto que se manifestó de diversos modos: en abrazos al Instituto, en clases públicas, marchas, blogs y conferencias de prensa, todo lo cual involucró a actores provenientes de diversos ámbitos hasta llegar a la judicialización. Así, ante la ausencia de la posibilidad de discutir y dirimir la forma en que desde el Estado son pensados, clasificados y medidos ciertos fenómenos sociales como la pobreza, la discusión se expandió a otras esferas, con otras reglas, otros formatos y otras dinámicas.

El presupuesto subyacente a la controversia cuyo foco estuvo puesto en lo metodológico, es que la realidad de la cosa mensurable, es decir, de la pobreza, es independiente del proceso de medición y, por ende, no es puesta en cuestión. En sintonía con esta percepción, en las controversias centradas en las mediciones, el disenso gira en torno a la fiabilidad de la operación aritmética, de modo que se relega o posterga una vez más la discusión profunda no sólo acerca de los métodos de medición, sino también acerca de la definición y conceptualización de la pobreza.

En síntesis, la reconstrucción de este proceso a partir del modelo de espacios controversiales, permitió la detección de los focos de las controversias 
y, así, periodizarlas. Por un lado, se pudo dar cuenta de los presupuestos compartidos, es decir, de las continuidades que subyacen a la efervescencia de las controversias y al cambio de actores; dichos presupuestos se encuentran vinculados a las estrategias de medición impuestas por organismos multilaterales de crédito, como el Banco Mundial. Al mismo tiempo, se detectaron ciertas rupturas (cambios de foco) que oscilaron de discusiones de corte metodológico a controversias sobre los resultados. En este sentido, el pasaje de las controversias al conflicto, y los acuerdos tácitos, se plasman en la "superficialización" del foco de la última controversia en torno a los resultados, lo que evidencia un proceso de estancamiento que se consolidó con la etapa de conflicto.

\section{Bibliografía}

Agis, Emmanuel, Carlos Cañete y Demian Panigo, 2011, El impacto de la asignación universal por hijo en Argentina, documento de trabajo, CEIL-PIETTE CONICET.

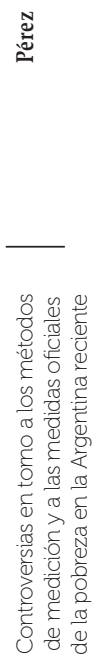

Alkire, Sabina y James Foster, 2008, “Counting and Multidimensional Poverty Measurement", Working Paper, núm. 7 y núm. 32, Oxford Poverty and Human Development Initiative.

Alkire, Sabina y Emma Santos, 2010, “Acute Multidimensional Poverty: A New Index for Developing countries”, OPHI, Working Paper núm. 38, University of Oxford.

Armatte, Michel, 2000, “Sociología e historia de la modelización estadística", Empiria, Revista de Metodología de Ciencias Sociales, núm. 3, pp. 11-34.

Boltvinik, Julio, 1992, “El método de medición integrada de la pobreza. Una propuesta para su desarrollo", Comercio Exterior, vol. 42, núm. 4, abril de1992, pp. 354-365.

Boltvinik, Julio, 2005, Ampliar la mirada. Un nuevo enfoque de la pobreza y el florecimiento humano, tesis doctoral, Centro de Investigaciones y Estudios Superiores en Antropología Social-Occidente.

Dascal, Marcelo, 1995, “Epistemology, controversies, and Pragmatics”, disponible en http:/ / www.focusing.org/apm_papers/dascal2.html

Dascal, Marcelo, 2006, "Compreendendo as controvérsias", Interpretação e compreensão, São Leopoldo, Unisinos. 
Desrosières, Alan, 1999, "The History of statistics as a genre: styles of writing and social uses", conferencia presentada en la Statistical Intenationalism, State Practices, and national traditions: progress report and prospects in the history of statistics, septiembre, Montreal, Canadá, University of Quebec, pp. 22-23.

Desrosières, Alan, 2004, La política de los grandes números. Historia de la razón estadística, España, Melusina.

Engelhardt, Tristam, 1981, Scientific Controversies (Case studies in the resolution and closure of disputes in science and technology), Cambridge University Press.

Esquivel, Juan Cruz, 2000, “Iglesia católica, política y sociedad: un estudio de las relaciones entre la elite eclesiástica argentina, el Estado y la sociedad en perspectiva histórica", en Informe final del concurso: Democracia, derechos sociales y equidad; y Estado, política y conflictos sociales, Programa Regional de Becas CLACso, Buenos Aires.

Fiscalía de Investigaciones Administrativas de Nación, 2007a, Informe preliminar causa No 5197/07(12441).

Fiscalía de Investigaciones Administrativas de Nación, 2007b, Aporte de prueba y documentación en los autos núm. 5197/07 caratulados “Moreno Guillermo y otros/violación de secretos particulares" y autos núm. 14463/07 caratulados "Paglieri Beatriz s/falsedad ideológica".

Hacking, Ian, 2001, ¿La construcción social de qué?, Barcelona, Editorial Paidós.

Instituto Nacional de Estadísticas y Censos, 1985, Métodos alternativos para medir la evolución del tamaño de la pobreza, Documento de trabajo núm. 6.

Instituto Nacional de Estadísticas y Censos, 2001, Índice de precios al consumidor base 1999. Metodología núm. 13.

Instituto Nacional de Estadísticas y Censos, 2003a, La encuesta permanente de hogares en Argentina, 2003, Instituto Nacional de Estadística y Censos. Encuesta permanente de hogares (EPH).

Instituto Nacional de Estadísticas y Censos, 2003b, Acerca del método utilizado para la medición de la pobreza en Argentina.

Instituto Nacional de Estadísticas y Censos, 2004, Incidencia de la pobreza y de la indigencia aglomerados de la encuesta permanente de hogares con medición puntual. 
Instituto Nacional de Estadísticas y Censos, 2005, Incidencia de la pobreza y de la indigencia aglomerados de la encuesta permanente de hogares con medición puntual.

Instituto Nacional de Estadísticas y Censos, 2007, Lineamientos del Programa Estadístico Nacional 2007-2011.

Mallimaci, Fortunato, 2005, "Catolicismo y política en el gobierno de Kirchner", América Latina Hoy, vol. 41, Salamanca, Universidad de Salamanca, pp. 57-76.

Nudler, Óscar, 2009, Espacios controversiales. Hacia un modelo de cambio filosófico y científico, Buenos Aires, Prometeo.

Nussbaum, Martha y Amartya Sen (comp.), 1996, La calidad de vida, México, Fondo de Cultura Económica.

Nussbaum, Martha, 2001, Women and Human Development, Cambridge University Press.

Nussbaum, Martha, 2003, "Capabilities as fundamental entitlements: Sen and Social Justice", Feminist Economics 9 (2/3), pp. 33-59.

Otero, Hernán, 2006, Estadística y nación. Una historia conceptual del pensamiento censal de la Argentina moderna 1869-1914, Buenos Aires, Prometeo editorial.

Otero, Hernán, 2011, “El concepto de población en el sistema estadístico de Argentina, 1869-2001”, Estatistica e Sociedade, núm. 1, Porto Alegre, pp. 7-25

Pardo Abril, Neira, 2008, “La representación de lo mensurable sobre la pobreza en la prensa colombiana", Discurso y Sociedad, 2(2), pp. 394-421.

Pogge, Thomas y Sanjay Reddy, 2003, How not to count the poor, University of Columbia.

Ravallion, Martin, 2008, How not to count the poor? A response to Reddy and Pogge, Development Research Group, Banco Mundial.

Ravallion, Martin, 2008, "Which poverty line. Response to Reddy", One Pager núm. 53, International Poverty Centre.

Reddy, Sanjay, 2008, “Are estimates on Latin American poverty reliable?”, One Pager, núm. 52, International Poverty Centre. 
Roca, Emilia, 2011, “La Asignación Universal por Hijo. Extensión de las asignaciones familiares", Debate Público. Reflexión de Trabajo Social, pp. 30-43

Sen, Amartya, 1976, "Poverty: an ordinal approach to measurement", Econométrica, vol. 44, pp. 219-232.

Sen, Amartya, 1980, "Equality of what?", The tanner lectures on human values, Salt Lake City, S. McMurrin.

Sen, Amartya, [1981]1992, "Sobre conceptos y medidas de pobreza", Comercio Exterior, vol. 42, núm. 4, México, pp.310-322.

Sen, Amartya, 1985, "A sociological approach to the measurement of poverty: a reply to professor Peter Townsend", Oxford Economic Papers, núm. 37.

Sen, Amartya, 1997, Bienestar, justicia y mercado, Barcelona, Paidós.

Sen, Amartya, 2000, Desarrollo y libertad, Madrid, Planeta.

Sen, Amartya, 2000b, Social exclusion: concept, application and scrutinity, master of Trinity College, Cambridge, Harvard University Social Development, Paper núm. 1, Office of Environment and Social Development Bank.

Torrado, Susana, 2006, Hogares y familia en América Latina, trabajo presentado en el II Congreso de la Asociación Latinoamericana de Población, Guadalajara, México.

Torrado, Susana, 2007, “El sistema estadístico nacional y la sociología: 50 años de experiencia", Revista Argentina de Sociología, vol. 5, núm. 9, Buenos Aires.

Whelan, Christopher T. y Brian Nola, 1996, "Measuring Poverty Using Income and Deprivation Indicators: Alternative Approaches", Journal of European Social Policy, vol. 6, núm. 3, pp. 225-240.

Recibido el 12 de noviembre de 2011

Aceptado el 15 de octubre de 2012 